\title{
Enhancement of Autophagy by Simvastatin through Inhibition of Rac1-mTOR Signaling Pathway in Coronary Arterial Myocytes
}

\author{
Yu-Miao Wei Xiang Li Ming Xu Justine M. Abais Yang Chen \\ Christopher R. Riebling Krishna M. Boini Pin-Lan Li Yang Zhang \\ Department of Pharmacology \& Toxicology, Medical College of Virginia Campus, Virginia \\ Commonwealth University, VA 23298
}

\author{
Key Words \\ Simvastatin $•$ Autophagy $\cdot \mathrm{mTOR} \cdot$ Coronary arterial myocyte $・$ Atherosclerosis
}

\begin{abstract}
Background/Aims: In addition to their action of lowering blood cholesterol levels, statins modulate biological characteristics and functions of arterial myocytes such as viability, proliferation, apoptosis, survival and contraction. The present study tested whether simvastatin, as a prototype statin, enhances autophagy in coronary arterial myocytes (CAMs) to thereby exert their beneficial effects in atherosclerosis. Methods and Results: Using flow cytometry, we demonstrated that simvastatin significantly increased the autophagsome formation in CAMs. Western blot analysis confirmed that simvastatin significantly increased protein expression of typical autophagy markers LC3B and Beclin1 in these CAMs. Confocal microscopy further demonstrated that simvastatin increased fusion of autophagosomes with lysosomes, which was blocked by autophagy inhibitor 3-methyladenine or silencing of Atg7 genes. Simvastatin reduced mammalian target of rapamycin (mTOR) activity, which was reversed by Rac1-GTPase overexpression and the mTOR agonist phosphatidic acid. Moreover, both Rac1-GTPase overexpression and activation of mTOR by phosphatidic acid drastically blocked simvastatin-induced autophagosome formation in CAMs. Interestingly, simvastatin increased protein expression of a contractile phenotype marker calponin in CAMs, which was blocked by autophagy inhibitor 3-methyladenine. Simvastatin markedly reduced proliferation of CAMs under both control and proatherogenic stimulation. However, this inhibitory effect of simvastatin on CAM proliferation was blocked by by autophagy inhibitor 3-methyladenine or silencing of Atg7 genes. Lastly, animal experiments demonstrated that simvastatin increased protein expression of LC3B and calponin in mouse coronary arteries. Conclusion: Our results indicate that simvastatin inhibits the Rac1-mTOR pathway and thereby increases autophagy in CAMs which may stabilize CAMs in the contractile phenotype to prevent proliferation and growth of these cells.

Copyright (C) 2013 S. Karger AG, Basel
\end{abstract}

Yang Zhang, Ph.D

Department of Pharmacology \& Toxicology, Medical College of Virginia Campus,

Virginia Commonwealth University, Richmond, VA 23298 (USA)

Tel. +1 (804) 828-0738, Fax +1 (804) 828-4794, E-Mail yzhang3@vcu.edu 


\section{Introduction}

Autophagy (also referred to macroautophagy) is an evolutionarily conserved process that results in degradation of cytosolic components inside lysosomes, which are eventually degraded by lysosomal hydrolases. These degraded materials can be reused for metabolic pathways as well as protein and nucleic acid synthesis. Under normal conditions, autophagy is a life-sustaining mechanism for recycling cellular components, while in an adverse environment autophagy is thought to be a cell adaptation and defense [1,2]. During this process, nascent double-membraned autophagic vacuoles (autophagosomes) surround and sequester a cargo, such as protein aggregates, damaged organelles (e.g. mitochondria, peroxisomes, endoplasmic reticulum membrane), or pathogens [1, 2]. Late stage autophagosomes fuse with lysosomes to form single membrane bound autophagolysosomes, where the cargo is degraded by lysosomal cathepsins [1,2]. This degradation process regenerates metabolic precursor molecules, like amino acids and fatty acids, that can be reutilized for de novo synthesis of macromolecules and energy generation $[1,2]$. Accumulating evidence has indicated that the role of autophagy in the vascular smooth muscle cells (VSMCs) may be both protective and detrimental during atherosclerosis, depending on the status of autophagy or stage of atherosclerosis. The importance of increased VSMC proliferation in the growth of atherosclerotic plaques has been extensively studied in animal models as well as human vascular obstructive lesions [3-5]. Enhanced autophagy in VSMCs at the early developmental stage of atherosclerosis may have beneficial effects via inducing a phenotype switch of these cells into a more differentiated and contractile phenotype, thereby decreasing cell proliferation and preventing fibrosis [4]. On the other hand, the typical atherosclerotic lesion shows a necrotic core surrounded by a fibrous cap consisting of VSMCs in a collagenproteoglycan matrix and the disappearance of VSMCs from the fibrous cap could jeopardize plaque stability thereby evoking atherothrombosis, myocardial infarction or stroke [6, 7]. In this regard, excessive autophagy in VSMCs may have detrimental effects via autophagic or type II cell death increasing the instability of atherosclerotic plaques $[8,9]$. Therefore, there are great potential clinical implications in understanding the mechanisms underlying autophagic regulation of vascular homeostasis by pharmacological interventions.

Statins, inhibitors of 3-hydroxy-3-methylglutaryl coenzyme A (HMG-CoA) reductase, block de novo cholesterol biosynthesis and therefore are widely used to lower plasma cholesterol and treat atherosclerotic disease [10, 11]. However, recent observations demonstrate that statins have pleiotropic effects that are well beyond their lipid-lowering properties. It has been shown that statins have anti-inflammation and immunomodulatory properties, improve nitric oxide bioavailability, prevent endothelial dysfunction, and stabilize atherosclerotic plaques [11-13]. Inhibition of HMG-CoA reductase by statins attenuates cholesterol synthesis through inhibition of the mevalonate pathway, which is essential for the synthesis of isoprenoid intermediates and consequent protein prenylation [11]. Although cholesterol is one product of prenylation, this same pathway also promotes activation of small GTPases such as Rho, Ras, and Rac via their geranylgeranlyation and subsequent translocation to the cell membrane [11]. This membrane localization of small GTPases in turn favors GTP binding and thus GTPase activation. Recent studies have shown that statins could induce autophagy in various mammalian cells [14-16]. However, it remains unknown whether statins could enhance autophagy in VSMCs via reduction of small GTPase prenylation. The present study hypothesized that enhanced autophagy by statins is an important effector mechanism leading to their anti-proliferative effects on vascular smooth muscle.

To test this hypothesis, we treated coronary arterial myocytes (CAMs, i.e. VSMCs of coronary arteries) and mice with simvastatin, a clinically widely used statin, and observed the autophagosome formation and changes in the expression of differentiation markers. Our results indicate that simvastatin inhibits the Rac1-mTOR pathway and thereby increases the formation of autophagosomes in CAMs. We also demonstrated that this simvastatin-induced autophagy mediates the upregulation of calponin, a contractile phenotype marker, in CAMs 
and arterial wall of coronary arteries. These studies thus reveal a potential mechanism for the benefical action of statins and suggest that they may enhance autophagy via inhibition of the Rac1-mTOR pathway, thereby stabilizing VSMCs in a more contractile phenotype, preventing these cells from proliferation and growth.

\section{Materials and Methods}

Culture of mouse coronary arterial myocytes (CAMs)

Mouse CAMs were isolated from C57BL/6 mice as we previously described [17]. In brief, mice were deeply anesthetized with an intraperitoneal injection of pentobarbital sodium $(25 \mathrm{mg} / \mathrm{kg})$. The heart was excised with an intact aortic arch and immersed in a petri dish filled with ice-cold Krebs-Henseleit solution. A 25-gauge needle filled with Hanks' buffered saline solution was inserted into the aortic lumen opening while the whole heart remained in the ice-cold buffer solution. The opening of the needle was inserted deep into the heart close to the aortic valve. The needle was tied in place with the needle tip as close to the base of the heart as possible. The infusion pump was started with a $20 \mathrm{ml}$ syringe containing warm HBSS through an intravenous extension set at a rate of $0.1 \mathrm{ml} / \mathrm{min}$ for $15 \mathrm{~min}$. HBSS was replaced with warm enzyme solution $(1 \mathrm{mg} / \mathrm{ml}$ collagenase type $\mathrm{I}, 0.5 \mathrm{mg} / \mathrm{ml}$ soybean trypsin inhibitor, $3 \%$ bovine serum albumin (BSA), and $2 \%$ antibiotic), which was flushed through the heart at a rate of $0.1 \mathrm{ml} / \mathrm{min}$. Perfusion fluid was collected at 30-, 60-, and 90-min intervals. At 90 min, the heart was cut with scissors, and the apex was opened to flush out the cells that collected inside the ventricle. The fluid was centrifuged at $1000 \mathrm{rpm}$ for 10 min, the cell-rich pellets were mixed with the one of the media described below, and the cells were plated on $2 \%$ gelatin-coated six-well plates and incubated in $5 \% \mathrm{CO}_{2}$ at $37{ }^{\circ} \mathrm{C}$. Advanced Dulbecco's modified Eagle's medium (DMEM) with 10\% fetal bovine serum, 10\% mouse serum, and $2 \%$ antibiotics was used for isolated smooth muscle cells. The identification of CAMs was based on positive staining by anti- $\alpha$-actin antibody and the smooth muscle cell morphology. The medium was replaced 3 days after cell isolation and then once or twice each week until the cells grew to confluence. All studies were performed with cells of passage of 3-5.

\section{Reagents and treatment}

Simvastatin was activated by opening the lactone ring by dissolving in $95 \%$ ethanol and $0.1 \mathrm{~N} \mathrm{NaOH}$, heating at $50{ }^{\circ} \mathrm{C}$ for $2 \mathrm{~h}$, and neutralizing with $\mathrm{HCl}$ to $\mathrm{pH} 7.2$ as described previously [18, 19]. Cells were treated with simvastatin $(5 \mu \mathrm{M})$ for 18 hours in the present study if not otherwise mentioned. The dosage was chosen based on our observations that simvastatin induced submaximal activation of autophagy (Fig. 1). In some groups, cells were treated with vehicle (phosphate buffered saline, PBS) or simvastatin $(5 \mu \mathrm{M})$ in the presence or absence of 3-methyladenine (3-MA, $5 \mathrm{mM}$ ), or phosphatidic acid (PA, $10 \mu \mathrm{M}$ ) (all reagents were purchased from Sigma). Rapamycin (20 nM; Sigma) was used as a classical stimuli for autophagy. NSC 23766 (30 $\mu \mathrm{M}$, TOCRIS Biosciences) was used as specific Rac1 inhibitor. OxLDL (10 $\mu$ M; KALEN Biomedical) was used as proatherogenic stimuli to treat CAMs. Atg7 siRNA or mTOR siRNA was purchased from Santa Cruz or Cell Signaling, respectively. Transfection of siRNA was performed using the siLentFect Lipid Reagent (Bio-Rad) according to the manufacturer's instructions as we described previously [20]. The efficiency of gene silencing was assessed by Western blotting, and real-time PCR analysis.

\section{Flow cytometric detection of autophagosomes}

Autophagosome formation in cells was deteced using a CytoID Autopagy Detection Kit (Enzo, PA, USA) following manufacturer's instruction. The CytoID fluorescent reagents specifically detect the autophagic vacuoles formed during autophagy. Briefly, cells were trypsinized, spun down, and washed twice in phenol red-free RPMI 1640 with $2 \%$ fetal bovine serum (FBS). The cells were resuspended in $0.5 \mathrm{ml}$ of freashly diluted CytoID reagents and incubated at $37^{\circ} \mathrm{C}$ for 30 minutes. The CytoID fluorescence of cells was immediately analyzed by flow cytometry using a flow cytometer (GUAVA, Hayward, CA, USA). The percentage of cells with CytoID staining was used to represent the formation of autophagosomes.

Western blot analysis

Western blot analysis was performed as we described previously [21]. In brief, proteins from the CAMs were extracted using sucrose buffer containing protease inhibitor. After being boiled for 5 min at $95{ }^{\circ} \mathrm{C}$ in a 
$5 \times$ loading buffer, $30 \mu \mathrm{g}$ of total proteins were separated by a $12 \%$ sodium dodecyl sulfate-polyacrylamide gel electrophoresis (SDS-PAGE). The proteins of these samples were then electrophoretically transferred at $100 \mathrm{~V}$ for 1 hour onto a PVDF membrane (Bio-Rad, USA). The membrane was blocked with 5\% nonfat milk in Tris-buffered saline-Tween 20 and after washing, the membrane was probed with rabbit anti-LC3B (1:1000, Cell Signaling), rabbit anti-Beclin 1 (1:1000, Cell Signaling), rabbit anti-Atg7 (1:1000, Cell Signaling), or mouse anti-calponin (1:500, Santa Cruz), overnight at $4{ }^{\circ} \mathrm{C}$, followed by incubation with horseradish peroxidase-labeled IgG (1:5000). The immuno-reactive bands were detected by chemiluminescence methods and visualized on Kodak Omat X-ray films. Densitometric analysis of the images obtained from $\mathrm{X}$-ray films was performed using the Image J software $(\mathrm{NIH})$. The relative protein expression over $\beta$-actin was quantified for comparison.

\section{Nucleofection}

Transfection of cDNA plasmids was performed using a 4D Nucleofector X-Unit (Lonza, CA, USA) according to the manufacturer's instructions. GFP-LC3B plasmid was obtained from Origene. Plasmids containing cDNA encoding constitutive mutant Rac1 gene was used as we previously described [21]. Briefly, CAMs were tyrpsinized and centrifuged at $90 \times g$ for 10 minutes. The cell pellet was resuspended in 100 $\mu \mathrm{L}$ P1 Nucleofection solution (Lonza) for Nucleofection (with the program code CM137). The program was chosen based on the fact that Nucleofection efficiency was over $80 \%$ as analyzed by flow cytometry using control GFP plasmids. For each Nucleofection sample, $2 \mu \mathrm{g}$ plasmid DNA was added in $100 \mu \mathrm{L}$ P1 Nucleofection solution. After Nucleofection, cells were cultured in DMEM medium for 24 hours and then cells were ready for the treatments.

\section{Confocal microscopy of autophagolysosomal fusion in living CAMs}

For confocal analysis, cultured CAMs were transfected with GFP-LC3B plasmid vectors by Nucleofection as described above. Cells were grown on glass chamber slides, stimulated or unstimulated, and stained with Lysotracker Red DND99 (1 $\mu$ M, Invitrogen) in phosphate-buffer saline (PBS) for $15 \mathrm{~min}$. Then, the slides were washed and bathed in PBS and visualized through sequentially scanning on an Olympus laser scanning confocal microscope (Fluoview FV1000, Olympus, Japan). The colocalization efficiency was analyzed by the Image Pro Plus 6.0 software (Media Cybernetics, Bethesda, MD, USA) as we described previously [22].

\section{Rac1 activation assay}

A specific pull-down experiment was performed to determine Rac1 GTPase activation using an Rac1 activation assay kit (Millipore) as described [23]. Briefly, cells were lysed in $\mathrm{Mg}^{2+}$ lysis buffer (MLB). To pull down Rac1-GTP, $200 \mu \mathrm{g}$ of cell extract was incubated for one hour at $4^{\circ} \mathrm{C}$ with $10 \mu \mathrm{g}$ of GST-PBD agarose beads (a fusion protein of glutathione S-transferase and p21-binding domain conjugated with agarose beads). The pelleted beads were washed three times with $0.5 \mathrm{~mL}$ of MLB, resuspended in $25 \mu \mathrm{L}$ of $2 \times$ Laemmli reducing sample buffer, and boiled for 5 minutes. The sample mixtures were then loaded on $12 \%$ SDS-PAGE gels. The bound active GTP-Rac1 molecules in the beads and total Rac1 in the cell lysates were analyzed by Western blot using a mouse anti-Rac1 monoclonal antibody (1:500 dilution) (Millipore).

\section{Mammalian Target of Rapamycin (mTOR) activity assay}

The activity of mTOR in CAMs was evaluated using the Kinase-linked immunosorbent assay (K-LISA ${ }^{\mathrm{TM}}$ ) mTOR activity kit (Calbiochem), which is an ELISA-based activity assay that utilizes a p70S6K-GST fusion protein as a specific mTOR substrate. The assay was performed following the manufacturer's procedure.

\section{Proliferation assay}

The proliferation of CAMs was quantified using a Aqueous One Solution Cell Proliferation Assay kit (Promega) following the manufacturer's instruction. Cells were cultured in a 96-well plate 2,000 cells per well) with or without treatment as indicated. After 48 hours, cells were incubated with $20 \mu \mathrm{L}$ Aqueous One Solution for $60 \mathrm{~min}$ at $37^{\circ} \mathrm{C}$. Then the absorbance (at $490 \mathrm{~nm}$ ) of each sample was measured using a microplate reader. Calibration curves showed the fluorescence reading to be proportional to the cell number. The proliferation rate was obtained by calculating the fold change in the cell number of each sample before and after 48-hour incubation. 
Fig. 1. Simvastatin induces autophagy in CAMs. (A) and (B) Mouse CAMs were treated with vehicle control (Ctrl), simvastatin (Simva, 0-20 $\mu \mathrm{M}$ ), or rapamycin (Rapa, $20 \mathrm{nM}$ ) for 18 hours. Then autophagy induction was detected by flow cytometry analysis of cells incubated with CytoID green fluorescent probes which detect autophagic vacuoles. Panel A shows a representative histogram of flow cytometry analysis. Panel B shows the summarized data showing the percentage of cells that were positive for the CytoID fluorescence $(n=4)$. (C) Time course of simvastatininduced autophagy in CAMs $(n=5)$. (D) Representative Western blot gel documents showing the effect of simvastatin (5 $\mu \mathrm{M}$ for 18 hours) on LC3B, Beclin1, Atg7 and $\beta$-actin expression in CAMs. (E) Summarized data of Western blots $(n=4)$. * $\mathrm{P}<0.05$ vs. Ctrl.

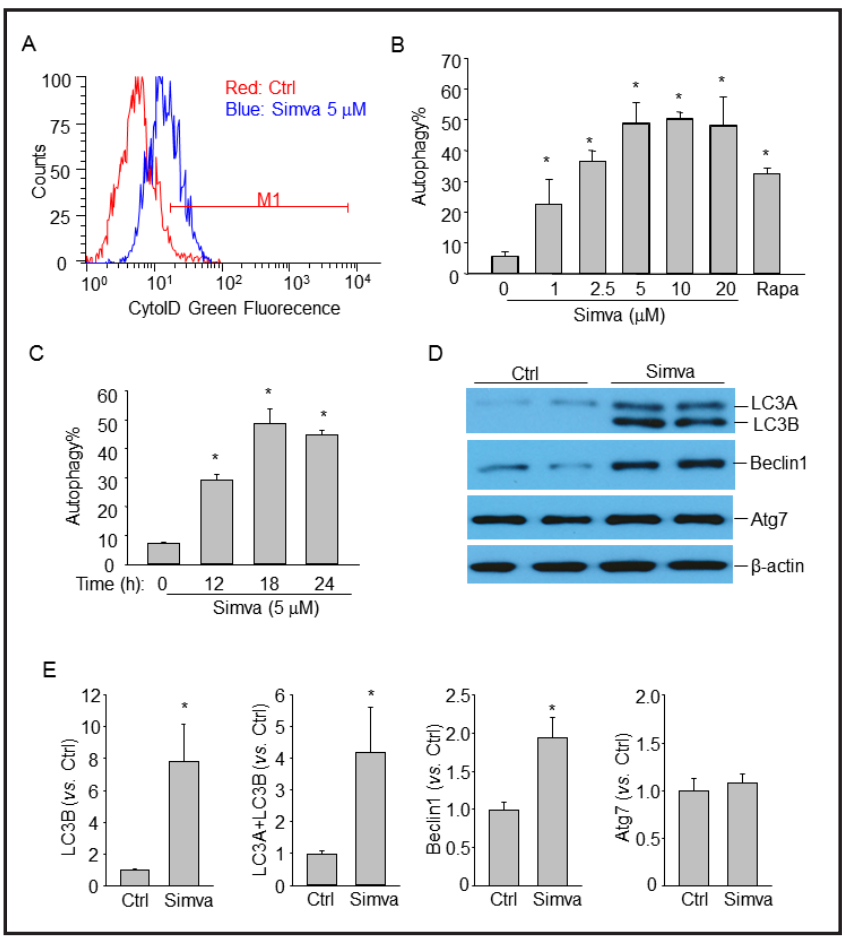

Animal procedures

C57BL/6J wild-type mice (6 weeks of age) (The Jackson Laboratory) were used in the present study. The mice were intragastrically fed simvastatin $20 \mathrm{mg} / \mathrm{kg} / \mathrm{d}$ for 2 weeks with or without mevalonate (intraperitoneal injection, $20 \mathrm{mg} / \mathrm{kg} / \mathrm{d}$ ). Control mice were intraperitoneally injected the same volume of saline. All the mice were sacrificed by cervical dislocation under ether anesthesia. The hearts with coronary artery were obtained and frozen in liquid nitrogen for preparation of frozen section slides. All protocols were approved by the Institutional Animal Care and Use Committee of Virginia Commonwealth University. All animals were provided standard rodent chow and water ad libitum in a temperature-controlled room.

\section{Immunofluorescent staining and confocal microscopic analysis}

The expression of LC3B and calponin in the coronary arterial wall were detected as described previously $[24,25]$. Briefly, the mouse hearts were frozen in Tissue-Tek OCT, cut by cryostat into $10 \mu \mathrm{m}$ sections and mounted on Superfrost/Plus slides. After fixation with acetone, the slides were incubated with goat anti- $\alpha$-smooth muscle actin antibody (1:50, Santa Cruz) and rabbit anti-LC3B (1:50, Cell Signaling) or mouse anti-calponin (1:50, Santa Cruz) overnight at $4^{\circ} \mathrm{C}$. After incubation with primary antibodies, the slides were washed and labeled with the corresponding Alexa Fluor-488 and Alexa Fluor-555 conjugated secondary antibodies (1:200, Invitrogen). Then, the slides were washed, mounted, and subjected to confocal microscopic analysis (Fluoview FV1000, Olympus, Japan).

\section{Statistics analysis}

Data are presented as means \pm SEM. Significant differences between and within multiple groups were examined using one-way ANOVA test followed by Duncan's multiple-range test. A Students' t test was used to detect significant difference between two groups. The statistical analysis was performed by SigmaStat 3.5 software (Systat Software). $\mathrm{P}<0.05$ was considered statistically significant.

\section{Results}

Simvastain induces autophagy in CAMs

To examine the possibility that stains induce autophagy in CAMs, we monitored autophagy in live cells by flow cytometry using a novel dye that exhibits bright green 
Fig. 2. Simvastatin increases autophagolysosome formation in CAMs. (A) Mouse CAMs were transfected with GFP-LC3B cDNA plasmids, stimulated with simvastatin (Simva, $5 \mu \mathrm{M}$ ) and stained with lysotracker red DND-99. Representative images show the colocalization (yellow) of autophagosomes (green) and lysosomes (red). Each image includes an enlarged view of area of interest (AOI). Some groups of CAMs were pretreated with 3-MA or transfected with Atg7 siRNA (Atg7si) and then stimulated with simvastatin. (B) Summarized data show the colocalization coefficiency (n=4). (C) Effect of 3-MA or Atg7 siRNA on simvastatin-induced autophagy in CAMs using Cyto-ID probes $(\mathrm{n}=4){ }^{*} \mathrm{P}<0.05$ vs. Ctrl; \# $\mathrm{P}<0.05$ vs. Simva.

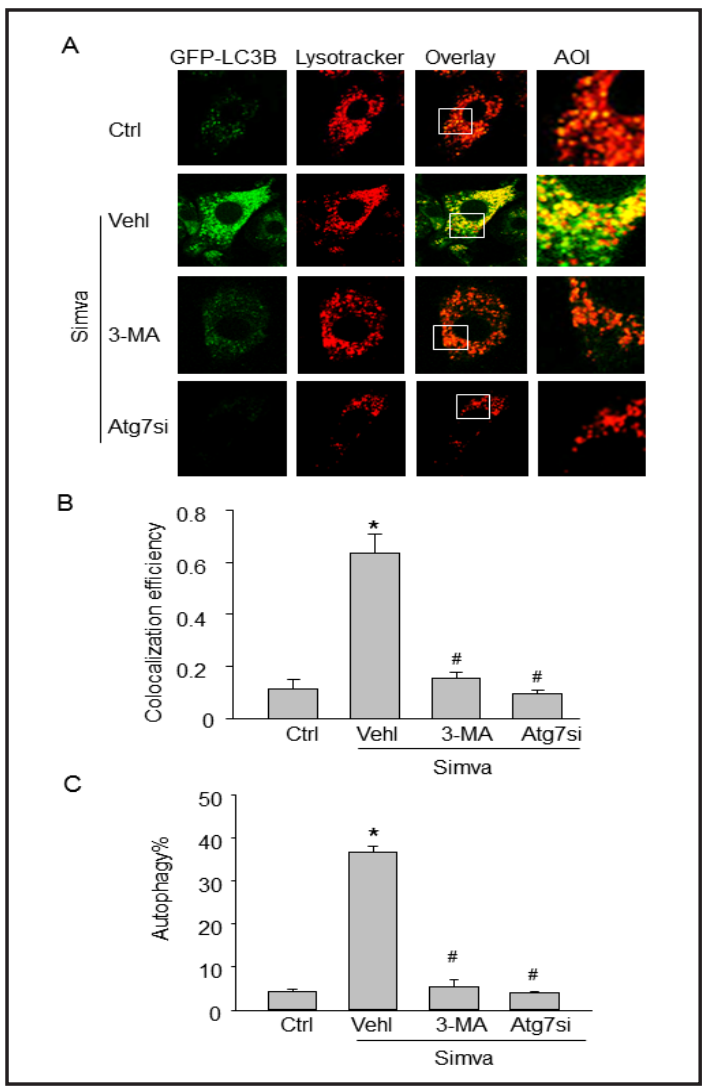

fluorescence when selectively labeling autophagic vacuoles including pre-autophagosomes, autophagosomes, and autophagolysosomes. Recently, this assay has been demonstrated to be a rapid and quantitative approach to monitor autophagy in live cells [26]. A representative flow cytometry histogram for CytoID fluorescence in CAMs is shown in Figure 1A. Simvastatin significantly increased CytoID fluorescence intensity suggesting more autophagic vacuoles were detected in simvastatin-treated CAMs. To quantify the potency of simvastatin-induced autophagy, we summarized the percentage of CytoID-positive cells in Figure 1B. As shown, simvastatin dose-dependently increased the percentage of CytoID-positive cells. Rapamycin, an autophagy inducer, was used as a positive control. As shown in Figure 1C, simvastatin at submaximal dose $(5 \mu \mathrm{M})$ time-dependently induced autophagy in CAMs which peaked at 18 hours after treatment. This dosage ( $5 \mu \mathrm{M}, 18$ hours) was chosen for the rest of experiments and our observations also showed that simvastatin at this dosage did not significantly induced morphologic signs of cytotoxicity in CAMs.

Induction of autophagy by simvastatin was also examined biochemically by detecting the protein expression of autophagy related genes by Western blot analysis (Fig. 1D and 1E). Coinciding to flow cytometric results, simvastatin markedly induced increases in LC3A, LC3B and Beclin1 expression but did not affect Atg7 expression.

Simvastatin induces fusion of autophagosomes with lysosomes in living CAMs

To directly investigate the fusion of autophagosomes with lysosome dynamically in living cells, we introduced GFP-LC3B vector into CAMs to express LC3B proteins that are located to autophagosomes and lysosomes were monitored with Lysotracker Red. As shown in Figure 2A, simvastatin stimulation led to remarkable interaction or fusion of autophagosomes (GFP-LC3B) and lysosomes (Lysotracker Red) as shown by dramatic increases in yellow areas. The summarized colocalization coefficiency further confirms that simvastatin promotes lysosome fusion with autophagosomes in CAMs (Fig. 2B). However, such simvastatin-induced interaction or fusion of autophagosomes with lysosomes was 
Fig. 3. Simvastatin induces autophagy in CAMs via inhibition of Rac1 and mTOR. (A) and (B) Mouse CAMs were treated with simvastatin (Simva, $5 \mu \mathrm{M}$ ) in the presence or absence of mevalonate (Mev, $10 \mu \mathrm{M}$ ), farnesol (Far, $10 \mu \mathrm{M}$ ), or geranylgeraniol (Ger, $10 \mu \mathrm{M}$ ). Representative Western blot gel document and summarized data show the relative expression of GTP-bound active Rac1 or total Rac1. (C) and (D) Mouse CAMs were treated simvastatin (Simva, 5 $\mu \mathrm{M}$ ), or NSC (Rac1 inhibitor, $30 \mu \mathrm{M}$ ), or transfected with Rac1 cDNA and then treated with simvastatin. Panel $C$ shows the summarized data for the activity of mTOR. Panel D shows the summarized data for the percentage of cells with autophagy using Cyto-ID probes. ${ }^{*} \mathrm{P}<0.05$ vs. Ctrl; \# $\mathrm{P}<0.05$ vs. Simva $(\mathrm{n}=4)$.

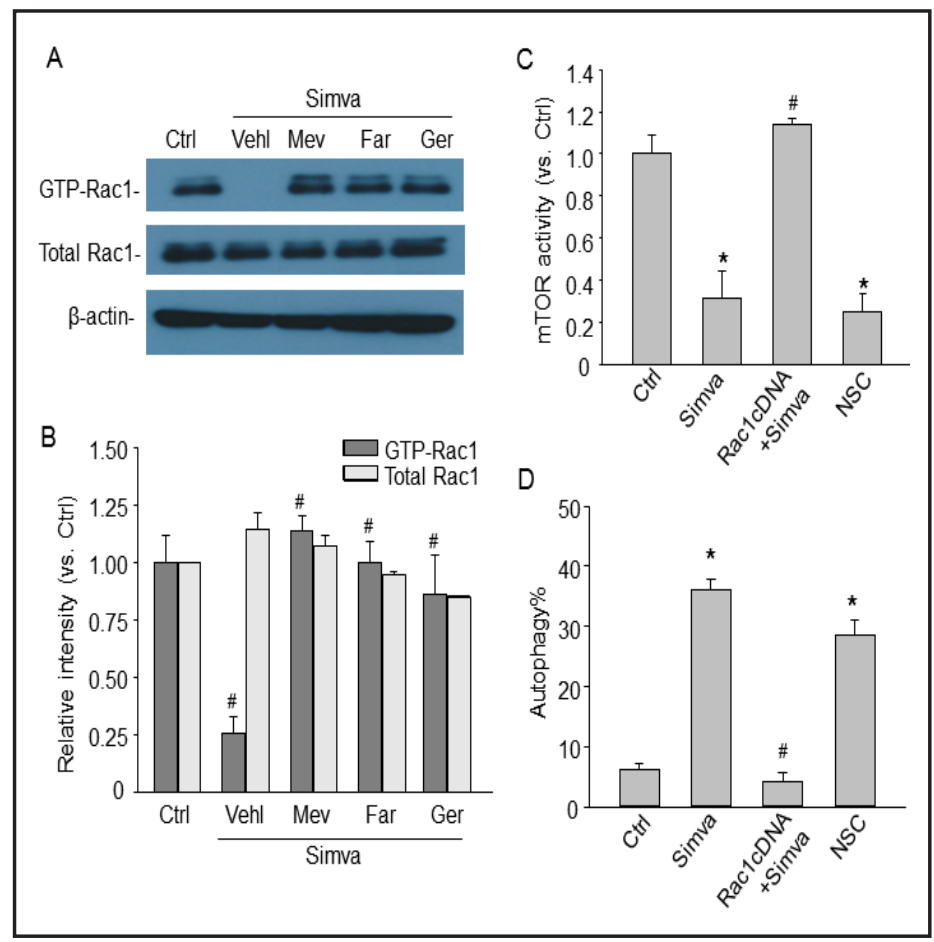

Fig. 4. Effect of mTOR activation and gene silencing on simvastatin-induced autophagy in CAMs. Mouse CAMs were unstimulated or stimulated with simvastatin (Simva) in the presence of mTOR activator phosphatidic acid (PA, $10 \mu \mathrm{M}$ ). Cells were also transfected with mTOR siRNA to knockdown the mTOR expression and then stimulated with phosphatidic acid. (A) Summarized data show the activity of mTOR. (B) Summarized data show the percentage of cells stained with CytoID fluorescence.* $\mathrm{P}<0.05$ vs. vehicle control; \# $\mathrm{P}<0.05$ vs simvastatin alone $(\mathrm{n}=4)$.

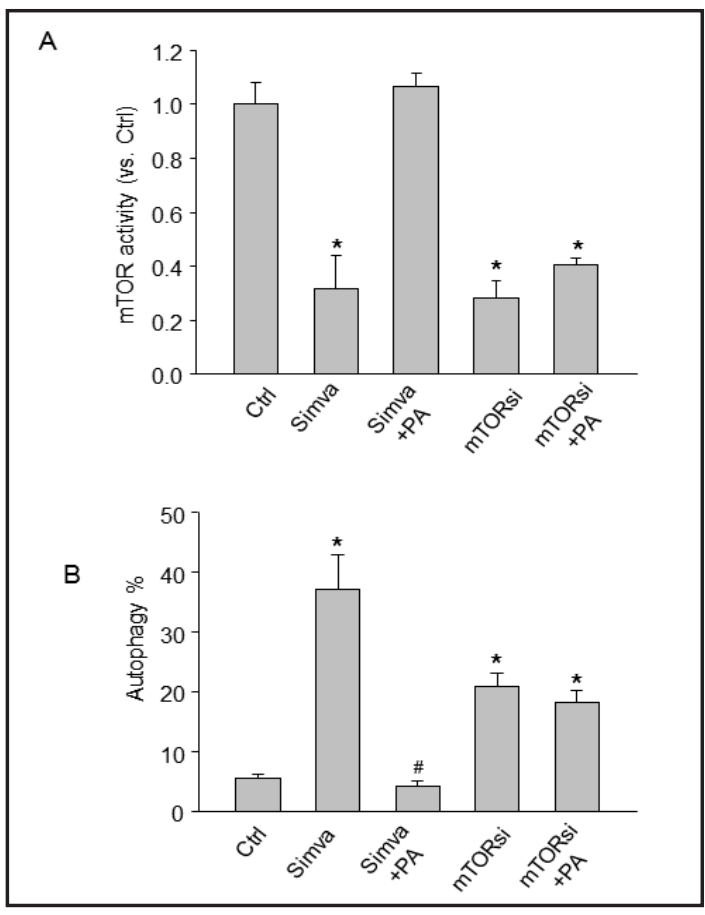

much less in CAMs treated with autophagy inhibitor 3-MA or Atg7 siRNA, which markedly reduced simvastatin-induced autophagy (Fig. 2C).

Simvastatin induces autophagy via inhibition of Rac1-mTOR signaling

To examine whether simvastatin inhibits Rac1-GTPase in CAMs, the active GTP-Rac1 was determined by a specific pull-down assay. As shown in Figure 3A and 3B, simvastatin inhibited Rac1 activity in CAMs and this simvastatin-induced Rac1 inhibition was reversed 
Fig. 5. Simvastatin-induced autophagy is assocatied with phenotypic changes of CAMs. CAMs were treated with simvastatin (Simva), rapamycin (Rapa), or 3-MA with simvastatin. (A) Representative gel document showing the effect of simvastatin on calponin expression. (B) Summarized data of Western blot. ${ }^{*} \mathrm{P}<0.05$ vs. Ctrl; \# $\mathrm{P}<0.05$ vs. Simva $(\mathrm{n}=6)$. (C) Summarized data showing the relative proliferation rate of CAMs treated with simvastatin or rapamycin in the presence or absence of 3-MA. * $\mathrm{P}<0.05$ vs. Ctrl; \# $\mathrm{P}<0.05$ vs. Simva; \$ $\mathrm{P}<0.05$ vs. Rapa ( $\mathrm{n}=5)$. (D) Summarized data show the effects of simvastatin on oxLDL-induced proliferation in CAMs with or without pretreatment of 3-MA or Atg7siRNA. * $\mathrm{P}<0.05$ vs. Ctrl; \# $\mathrm{P}<0.05$ vs. oxLDL; $\$ \mathrm{P}<0.05$ vs. Simva+oxLDL $(\mathrm{n}=5)$.

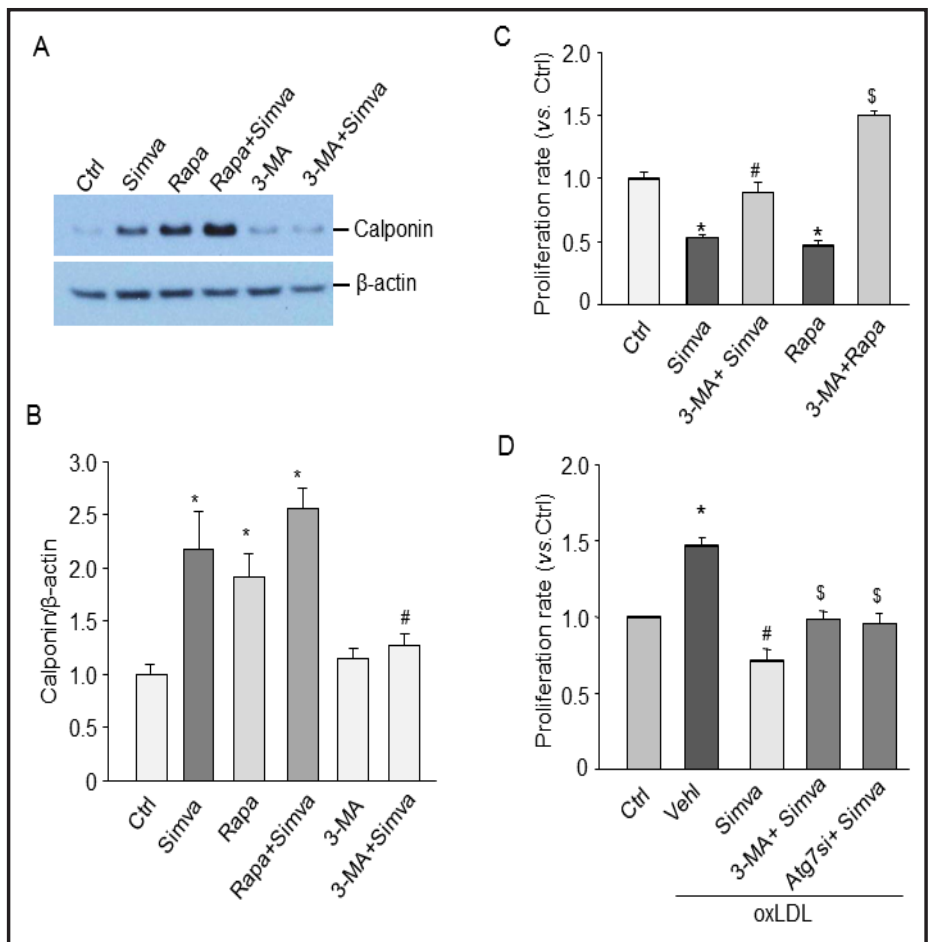

by rescuing mevalonate pathway with supplementation of mevalonate or rescuing isoprenylation using farnesol or geranylgeraniol.

To explore whether inhibition of Rac1-GTPase by simvastatin results in mTOR inhibition-induced autophagy, the effects of Rac1 overexpression on simvastatin-induced mTOR inhibiton and autophagy were examined. Rac 1 activity significantly increased in CAMs transfected with Rac 1 cDNA (data not shown). As shown in Figure 3C, both simvastatin and Rac1 inhibitor NSC blocked mTOR activity, however, overexpression of Rac1 reversed the inhibitory effect of simvastatin on mTOR activity. As shown in Figure 3D, Rac1 overexpression blocked simvastatin-induced autophagy. Inhibition of Rac1 by NSC mimicked the effect of simvastatin on autophagy induction in CAMs. Simvastatin-induced autophagy was blocked by supplementation of mevalonate in CAMs (6.73 $1.11 \%$; data were not shown as a bar). Taken together, these results indicate that the inhibition of Rac1 by simvastatin results in mTOR inhibition and induction of autophagy in CAMs.

\section{Effect of mTOR activation on simvastatin-induced autophagy}

To further examine whether inhibition of mTOR activity is involved in simvastatininduced autophagy, an mTOR agonist phosphatidic acid (PA) was added to the culture medium and its effect on the extent of autophagy induction was investigated. As shown in Figure 4A, mTOR agonist phosphatidic acid completely restored the mTOR activity in CAMs treated with simvastatin. mTOR activity was markedly reduced in CAMs with mTOR gene silencing, but was not significantly increased by phosphatidic acid. Consistently, as shown in Figure 4B, activation of mTOR by phosphatidic acid abolished simvastatin-induced autophagy and mTOR silencing alone induced autophagy. Thus, these results indicate that simvastatin-induced autophagy is associated with its inhibitory effect on mTOR activity.

\section{Inhibition of autophagy blocks simvastatin-induced phenotypic changes of CAMs}

To examine whether simvastatin-induced autophagy plays a role in differentiation by switching the CAMs from a contractile phenotype to a more proliferative phenotype, we measured the expression of calponin, a smooth muscle contractile marker, and the cell proliferation rate. As shown in Figures $5 \mathrm{~A}$ and $5 \mathrm{~B}$, simvastatin increased calponin 
Fig. 6. Simvastatin increase LC3B and calponin expression in coronary arteries. Mice were intragastrically fed simvastatin for two weeks with or without mevalonate. Frozen sections of mouse hearts were stained with Alexa488-anti- $\alpha$-smooth muscle actin $(\alpha-S M A)$ and Alexa555-anti-LC3B or Alexa555-anticalponin antibodies. Displayed are representative confocal microscopic images showing the expression of LC3B (A) or calponin (B) in coronary arteries $(n=5)$.

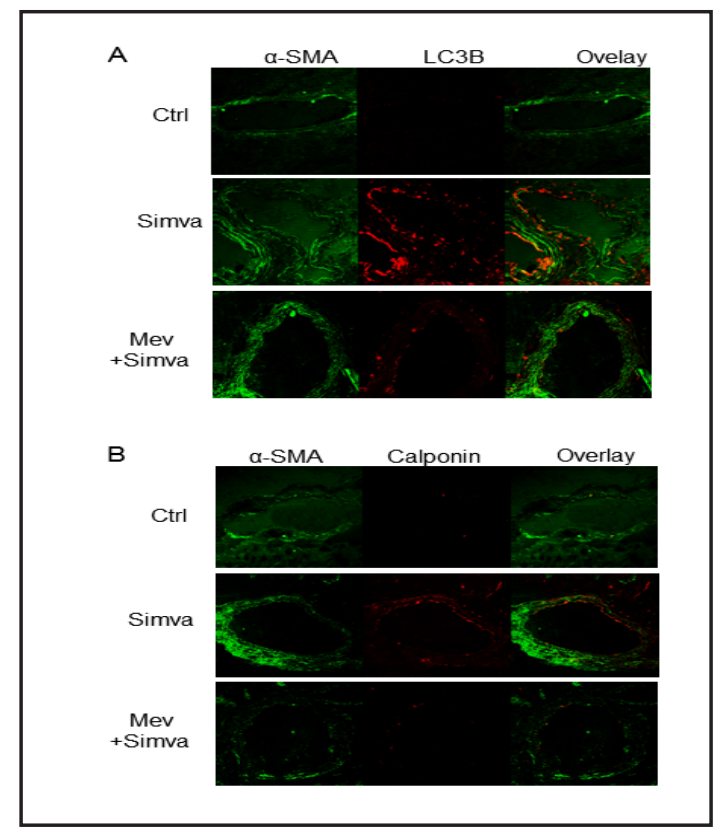

expression suggesting that simvastatin could stabilize CAMs to a contractile status. Similar to simvastatin, rapamycin increased calponin expression in CAMs. Further, the simvastatininduced calponin expression was blocked by autophagy inhibitor 3-MA. As shown in Figure 5C, simvastatin or rapamycin significantly decreased the proliferation rate of CAMs and these inhibitory effects of simvastatin or rapamycin were reversed by 3-MA. There was no cell death observed during the treatment of the cells with simvastatin or rapamycin indicating that the cell death is not involved in decreased cellular proliferation in our experimental settings. As shown in Figure 5D, simvastatin significantly inhibited oxLDLinduced proliferation in CAMs, which were partially restored by 3-MA or Atg7 gene silencing. Taken together, these results suggest that enhanced autophagy by simvastatin is involved in the stabilization of CAMs to a contractile phenotype under both control condition and proatherogenic stimulation.

\section{Simvastatin increases LC3B and calponin expression in mouse coronary artery}

To examine whether simvastatin-induced autophagy is implicated in in vivo phenotype switching, we treated mice with vehicle or simvastatin and stained frozen sections from mouse hearts with LC3B or calponin together with $\alpha$-smooth muscle actin ( $\alpha$-SMA). As shown in Figure 6, LC3B and calponin expression level was much higher in coronary arterial walls of simvastatin-treated mice compared to those from vehicle-treated control mice. Supplementation of mice with mevalonate blocked this effect of simvastatin on LC3B and calponin.

\section{Discussion}

The present study for the first time demonstrated that simvastatin increased autophagy in CAMs and in the arterial wall of coronary arteries via inhibition of the mevalonate pathway and of small GTPase Rac1. Notably, inhibition of Rac1 by simvastatin would also predict inhibitory effects on mTOR, a mammalian target of rapamycin associated with induction of autophagy. Our data thus suggest that inhibition of the Rac1-mTOR pathway and consequent enhancement of autophagy are important determinants of vascular smooth muscle protection by statins.

The hallmark of autophagy is the formation of the autophagosome, followed by its fusion with a lysosome, termed autophagolysosome. Microtubule-associated protein 1 light 
chain (LC3) exists in the cytoplasm in a soluble form (LC3A). A phosphatidylethanolamine (PE)-conjugated form of LC3 (LC3B) is associated with the phagophore and the inner autophagosomal membrane and is a highly specific marker for these structures [27]. Upon fusion with the lysosome, LC3B on the inner autophagosomal membrane is degraded [28]. Several recent studies have shown that statins induced formation of autophagosomes in mammalian cells $[14,15]$. For instance, cerivastatin and simvastatin induced autophagy in human rhabdomyosarcoma cells and atorvastatin increased autophagy in PC3 cells $[14,15]$. More recently, simvastatin was demonstrated to increase autophagy in human airway smooth muscle cells [16]. By using a specific fluorescent probe for autophagic vacuoles and detecting LC3B expression, we demonstrated for the first time that simvastatin enhances autophagy by increasing the formation of autophagosomes in primary culture of CAMs. Further, simvastatin increased autophagsosome fusion with lysosomes indicating formation of autophagolysosomes. Such simvastatin-induced increases in the formation of autophagsome and autophagolysosomes was blocked by 3-MA, an inhibitor of autophagosome formation. Thus, our data reveal that the inhibitory action of simvastatin on the HMG-CoA reductasemevalonate pathway is also associated with autophagy pathway.

The present study further demonstrated that the small GTPase Rac1 regulates mTORmediated autophagy, which links the inhibitory action of simvastatin on Rac1 activity to the mTOR-mediated autophagy pathway. Protein isoprenylation enables proper subcellular localization and trafficking of intracellular proteins [29]. Statins, via inhibition of the mevalonate pathway and thus the subsequent synthesis of isoprenoids and prenlylation action, facilitate accumulation of the inactive, small GTPase in the cytoplasm. It has been well documented that statins can inhibit the mevalonate pathway resulting in inhibition of Rac1 activity in vascular cells [25, 30, 31]. In the present study, we found that activation of Rac1 by overexpression of Rac1 or restoration of mevalonate pathway by supplementation of mevalonate prevented simvastatin-induced autophagy in CAMs, suggesting that Rac1 is an upstream signaling molecule controlling mTOR activity and its regulation of autophagy. Consistently, a recent study has demonstrated that Rac1 can regulate the activity of mTOR and control cellular size in mouse embryonic fibroblasts and lymphocytes [32]. Rac1 may directly bind to mTOR to mediate its localization at specific membranes [32]. In addition to Rac1, other small GTPases such as Rheb have been shown to be direct upstream activation factor of mTOR [33]. Thus, in line with these previous findings, our data support the view that simvastatin inhibits lipid-anchoring of the small G protein Rac1 to perturb mTOR signaling and thereby induce autophagy in CAMs.

The present study further revealed that inhibition of the mTOR signaling pathway is indeed involved in the autophagy induced by simvastatin. mTOR plays a central role in the processes of the autophagy pathway [34]. MTOR is a serine/threonine protein kinase that regulates many cellular activites including autophagy, proliferation, survival and metabolism in mammalian cells [34]. Activation of mTOR signaling is implicated in diseases such as cancer and tissue hypertrophy and inhibitors of mTOR signaling are used clinically to inhibit cancer growth and prevent graft rejection and restenosis after coronary angioplasty [35]. In the cardiovascular system, mTOR is the most important negative regulator of autophagy $[1,36]$. In the present study, we found that simvastatin inhibited mTOR activity and direct activation of mTOR by its agonist phosphatidic acid abolished simvastatin-induced autophagy. Our findings are consistent with recent studies showing that simvastatin inhibits mTOR and its substrate S6K in glioma cells [37] and blocks mTOR-mediated Go(s) translation in endothelial cells [38]. To our knowledge, the present study provided the first evidence that simvastatininduced autophagy is mediated by mTOR inhibition.

Autophagy represents an adaptive strategy by which cells survive bioenergetic stress or other pathological circumstances. Although excessive autophagy can cause cell death, enhanced and controlled autophagy is protective by providing essential substrates during starvation and by removing damaged materials. In the cardiovascular system, especially in heart ischemia and atherosclerosis, autophagy plays an essential role in the homeostasis of cardiovascular cell metabolism and regeneration [2]. In the mature vasculature, vascular 
smooth muscle exhibits a contractile or differentiated status characterized by the expression of contractile markers such as calponin. However, in a pathological status, such as in progressive atherosclerosis or restenosis after coronary angioplasty, VSMCs are prone to proliferation, migration and synthesis of extracellular matrix components [39, 40]. Recent studies found that rapamycin and atherosclerotic risk factors such as adipokine adiponectin can increase the contractile protein expression and induce VSMC differentiation, which are associated with enhanced autophagy signaling [41-43]. A recent study by Nakai et al. found that in Atg5-deficient mice, the cardiac phenotype and contractile function were severely impaired and they demonstrated that constitutive autophagy in the heart under baseline conditions is a homeostatic mechanism for maintaining cardiomyocyte size and global cardiac structure and function [36]. In the present study, we found that enhanced autophagy by simvastatin increased the expression of contractile phenotype marker calponin in CAMs and decreased their proliferation rate under both control and proatherogenic stimulation, whereas inhibition of autophagy by 3-MA reversed the effects of simvastatin on calponin expression and proliferation. These results suggest that simvastatin via autophagy signaling pathway can stabilize CAMs to a contractile status, preventing excessive proliferation and migration. To date, the mechanisms linking autophagy and VSMC differentiation are not clear. Our unpublished data demonstrated VSMC phenotype was switched from contractile to more proliferative when autophagic flux is abnormal due to loss of lysosomal acid sphingomyelinase indicating that normal autophagic flux is important for cell differentiation. More ongoing studies are currently conducted in our laboratories to decipher how autophagic flux is linked with cell differentiation.

Our animal study, for the first time, demonstrated that simvastatin increased autophagy and expression of contractile phenotype marker in mouse coronary arterial smooth muscle. Supplementation of mice with mevalonate blocked these effects of simvastatin. These data implicate that the Rac1-mTOR-mediated autophagy pathway modulates smooth muscle phenotype switching in vivo. Recent large scale clinical trials demonstrated that statins greatly decreased the cardiovascular events in patients with coronary heart diseases [44]. The beneficial effects have been largely attributed to their cholesterol depletion and antiinfllammatory actions. The present study revealed that autophagy via inhibition of the Rac1mTOR pathway may be one additional beneficial effect of statins on protecting vascular smooth muscle. It is plausible that at the early stage of the pathogenesis and progression of atherosclerosis, enhanced and controlled autophagy by statins may be a protective mechanism to prevent VSMC proliferation, but at the late stage of atherosclerosis, excessive and uncontrolled autophagy in VSMCs may cause autophagic cell death and plaque instability [45]. Our laboratories will further clarify how autophagy pathway mediates the beneficial action of statins in acute hypercholesterolemia and spontaneous atherosclerosis mouse models.

In summary, the present study demonstrated that simvastatin induced autophagy in primary cultured CAMs. This effect of simvastatin is associated with its inhibitory action on HMG-CoA reductase activity, which results in reduced prenylation and activation of Rac1GTPase leading to inhibition of the mTOR signaling pathway and consequent initiation of autophagsome formation. Moreover, simvastatin-induced autophagy plays an important role in the homeostasis of CAMs by maintaining their contractile status both in vitro and in vivo. These findings increase our understanding of the mechanisms underlying the pleiotropic effects of statins on vascular pathology, which are beyond their lipid-lowering properties.

\section{Acknowledgements}

This study was supported by grants from the National Institute of Health (HL057244, HL075316, and HL091464). 
Wei et al.: Simvastatin Enhances Autophagy via mTOR Pathway

\section{References}

1 Ryter SW, Lee SJ, Smith A, Choi AM: Autophagy in vascular disease. Proc Am Thorac Soc 2010;7:40-47.

2 Martinet W, Knaapen MW, Kockx MM, De Meyer GR: Autophagy in cardiovascular disease. Trends Mol Med 2007;13:482-491.

-3 Fuster JJ, Fernandez P, Gonzalez-Navarro H, Silvestre C, Nabah YN, Andres V: Control of cell proliferation in atherosclerosis: Insights from animal models and human studies. Cardiovasc Res 2010;86:254-264.

4 Lacolley P, Regnault V, Nicoletti A, Li Z, Michel JB: The vascular smooth muscle cell in arterial pathology: A cell that can take on multiple roles. Cardiovasc Res 2012;95:194-204.

5 Dzau VJ, Braun-Dullaeus RC, Sedding DG: Vascular proliferation and atherosclerosis: New perspectives and therapeutic strategies. Nat Med 2002;8:1249-1256.

6 Virmani R, Kolodgie FD, Burke AP, Farb A, Schwartz SM: Lessons from sudden coronary death: A comprehensive morphological classification scheme for atherosclerotic lesions. Arterioscler Thromb Vasc Biol 2000;20:1262-1275.

7 Clarke M, Bennett M: The emerging role of vascular smooth muscle cell apoptosis in atherosclerosis and plaque stability. Am J Nephrol 2006;26:531-535.

8 Jia G, Cheng G, Agrawal DK: Differential effects of insulin-like growth factor-1 and atheroma-associated cytokines on cell proliferation and apoptosis in plaque smooth muscle cells of symptomatic and asymptomatic patients with carotid stenosis. Immunol Cell Biol 2006;84:422-429.

-9 Martinet W, De Bie M, Schrijvers DM, De Meyer GR, Herman AG, Kockx MM: 7-ketocholesterol induces protein ubiquitination, myelin figure formation, and light chain 3 processing in vascular smooth muscle cells. Arterioscler Thromb Vasc Biol 2004;24:2296-2301.

10 Libby P, Ridker PM, Hansson GK: Inflammation in atherosclerosis: From pathophysiology to practice. J Am Coll Cardiol 2009;54:2129-2138.

11 Bu DX, Griffin G, Lichtman AH: Mechanisms for the anti-inflammatory effects of statins. Curr Opin Lipidol 2011;22:165-170.

12 Jacobson JR: Statins in endothelial signaling and activation. Antioxid Redox Signal 2009;11:811-821.

13 Mason RP, Walter MF, Jacob RF: Effects of hmg-coa reductase inhibitors on endothelial function: Role of microdomains and oxidative stress. Circulation 2004;109:II34-41.

14 Araki M, Motojima K: Hydrophobic statins induce autophagy in cultured human rhabdomyosarcoma cells. Biochem Biophys Res Commun 2008;367:462-467.

15 Parikh A, Childress C, Deitrick K, Lin Q, Rukstalis D, Yang W: Statin-induced autophagy by inhibition of geranylgeranyl biosynthesis in prostate cancer pc3 cells. Prostate 2010;70:971-981.

16 Ghavami S, Mutawe MM, Sharma P, Yeganeh B, McNeill KD, Klonisch T, Unruh H, Kashani HH, Schaafsma D, Los M, Halayko AJ: Mevalonate cascade regulation of airway mesenchymal cell autophagy and apoptosis: A dual role for p53. PLoS One 2011;6:e16523.

17 Xu M, Zhang Y, Xia M, Li XX, Ritter JK, Zhang F, Li PL: Nad(p)h oxidase-dependent intracellular and extracellular 02•- production in coronary arterial myocytes from cd38 knockout mice. Free Radic Biol Med 2012;52:357-365.

18 Gerson RJ, MacDonald JS, Alberts AW, Kornbrust DJ, Majka JA, Stubbs RJ, Bokelman DL: Animal safety and toxicology of simvastatin and related hydroxy-methylglutaryl-coenzyme a reductase inhibitors. Am J Med 1989;87:28S-38S.

19 Tawfik HE, El-Remessy AB, Matragoon S, Ma G, Caldwell RB, Caldwell RW: Simvastatin improves diabetesinduced coronary endothelial dysfunction. J Pharmacol Exp Ther 2006;319:386-395.

-20 Zhang G, Zhang F, Muh R, Yi F, Chalupsky K, Cai H, Li PL: Autocrine/paracrine pattern of superoxide production through nad(p)h oxidase in coronary arterial myocytes. Am J Physiol Heart Circ Physiol 2007;292:H483-495.

21 Yi F, Xia M, Li N, Zhang C, Tang L, Li PL: Contribution of guanine nucleotide exchange factor vav2 to hyperhomocysteinemic glomerulosclerosis in rats. Hypertension 2009;53:90-96.

-22 Xia M, Zhang C, Boini KM, Thacker AM, Li PL: Membrane raft-lysosome redox signalling platforms in coronary endothelial dysfunction induced by adipokine visfatin. Cardiovasc Res 2011;89:401-409.

-23 Yi F, Zhang AY, Janscha JL, Li PL, Zou AP: Homocysteine activates nadh/nadph oxidase through ceramidestimulated rac gtpase activity in rat mesangial cells. Kidney Int 2004;66:1977-1987. 
24 Zhang AY, Yi F, Zhang G, Gulbins E, Li PL: Lipid raft clustering and redox signaling platform formation in coronary arterial endothelial cells. Hypertension 2006;47:74-80.

25 Wei YM, Li X, Xiong J, Abais JM, Xia M, Boini KM, Zhang Y, Li PL: Attenuation by statins of membrane raftredox signaling in coronary arterial endothelium. J Pharmacol Exp Ther 2013;345:170-179.

26 Lee JS, Lee GM: Monitoring of autophagy in chinese hamster ovary cells using flow cytometry. Methods 2012;56:375-382.

27 Kabeya Y, Mizushima N, Ueno T, Yamamoto A, Kirisako T, Noda T, Kominami E, Ohsumi Y, Yoshimori T: Lc3, a mammalian homologue of yeast apg8p, is localized in autophagosome membranes after processing. EMBO J 2000;19:5720-5728.

-28 Xie Z, Klionsky DJ: Autophagosome formation: Core machinery and adaptations. Nat Cell Biol 2007;9:11021109.

29 Takai Y, Sasaki T, Matozaki T: Small gtp-binding proteins. Physiol Rev 2001;81:153-208.

- 30 Negre-Aminou P, van Erck M, van Leeuwen RE, Collard JG, Cohen LH: Differential effect of simvastatin on various signal transduction intermediates in cultured human smooth muscle cells. Biochem Pharmacol 2001;61:991-998.

31 Wassmann S, Laufs U, Baumer AT, Muller K, Konkol C, Sauer H, Bohm M, Nickenig G: Inhibition of geranylgeranylation reduces angiotensin ii-mediated free radical production in vascular smooth muscle cells: Involvement of angiotensin at1 receptor expression and rac1 gtpase. Mol Pharmacol 2001;59:646654.

32 Saci A, Cantley LC, Carpenter CL: Rac1 regulates the activity of mtorc1 and mtorc2 and controls cellular size. Mol Cell 2011;42:50-61.

33 Gulhati P, Bowen KA, Liu J, Stevens PD, Rychahou PG, Chen M, Lee EY, Weiss HL, O'Connor KL, Gao T, Evers BM: Mtorc1 and mtorc2 regulate emt, motility, and metastasis of colorectal cancer via rhoa and rac1 signaling pathways. Cancer Res 2011;71:3246-3256.

-34 Ravikumar B, Sarkar S, Davies JE, Futter M, Garcia-Arencibia M, Green-Thompson ZW, Jimenez-Sanchez M, Korolchuk VI, Lichtenberg M, Luo S, Massey DC, Menzies FM, Moreau K, Narayanan U, Renna M, Siddiqi FH, Underwood BR, Winslow AR, Rubinsztein DC: Regulation of mammalian autophagy in physiology and pathophysiology. Physiol Rev 2010;90:1383-1435.

35 Wang X, Proud CG: Mtorc1 signaling: What we still don't know. J Mol Cell Biol 2011;3:206-220.

-36 Nakai A, Yamaguchi O, Takeda T, Higuchi Y, Hikoso S, Taniike M, Omiya S, Mizote I, Matsumura Y, Asahi M, Nishida K, Hori M, Mizushima N, Otsu K: The role of autophagy in cardiomyocytes in the basal state and in response to hemodynamic stress. Nat Med 2007;13:619-624.

37 Misirkic M, Janjetovic K, Vucicevic L, Tovilovic G, Ristic B, Vilimanovich U, Harhaji-Trajkovic L, SumaracDumanovic M, Micic D, Bumbasirevic V, Trajkovic V: Inhibition of ampk-dependent autophagy enhances in vitro antiglioma effect of simvastatin. Pharmacol Res 2012;65:111-119.

38 Kou R, Shiroto T, Sartoretto JL, Michel T: Suppression of galphas synthesis by simvastatin treatment of vascular endothelial cells. J Biol Chem 2012;287:2643-2651.

39 Rzucidlo EM, Martin KA, Powell RJ: Regulation of vascular smooth muscle cell differentiation. J Vasc Surg 2007;45:A25-32.

-40 Orr AW, Hastings NE, Blackman BR, Wamhoff BR: Complex regulation and function of the inflammatory smooth muscle cell phenotype in atherosclerosis. J Vasc Res 2010;47:168-180.

-41 Ding M, Xie Y, Wagner RJ, Jin Y, Carrao AC, Liu LS, Guzman AK, Powell RJ, Hwa J, Rzucidlo EM, Martin KA: Adiponectin induces vascular smooth muscle cell differentiation via repression of mammalian target of rapamycin complex 1 and foxo4. Arterioscler Thromb Vasc Biol 2011;31:1403-1410.

-42 Martin KA, Rzucidlo EM, Merenick BL, Fingar DC, Brown DJ, Wagner RJ, Powell RJ: The mtor/p70 s6k1 pathway regulates vascular smooth muscle cell differentiation. Am J Physiol Cell Physiol 2004;286:C507517.

43 Brown DJ, Rzucidlo EM, Merenick BL, Wagner RJ, Martin KA, Powell RJ: Endothelial cell activation of the smooth muscle cell phosphoinositide 3-kinase/akt pathway promotes differentiation. J Vasc Surg 2005;41:509-516.

44 Biasucci LM, Biasillo G, Stefanelli A: Inflammatory markers, cholesterol and statins: Pathophysiological role and clinical importance. Clin Chem Lab Med 2010;48:1685-1691.

45 Martinet W, De Meyer GR: Autophagy in atherosclerosis: A cell survival and death phenomenon with therapeutic potential. Circ Res 2009;104:304-317. 